\title{
Medical students' mood adversely affected by COVID-19 pandemic: An interim analysis from the SPICE-19 prospective cohort study of 2075 medical students and interim foundation doctors
}

\section{Soham Bandyopadhyay ( $\sim$ soham.bandyopadhyay@yahoo.co.uk)}

Medical Sciences Division, University of Oxford, Oxford, UK https://orcid.org/0000-0001-6553-3842

loannis Georgiou

The School of Medicine, University of Aberdeen, Aberdeen, UK

Bibire Baykeens

Plymouth University Peninsula Schools of Medicine and Dentistry, UK

Conor S Gillespie

School of Medicine, University of Liverpool, Liverpool, UK

Marta de Andres Crespo

Medical Sciences Division, University of Oxford, Oxford, UK

Mohammad Talha Bashir

The School of Medicine, University of Aberdeen, Aberdeen, UK

Ashok Handa

Nuffield Department of Surgical Sciences, University of Oxford, Oxford, UK

Kate E A Saunders

Department of Psychiatry, Warneford Hospital, University of Oxford, Warneford Lane, Oxford, UK

\section{Research Article}

Keywords: Coronavirus, COVID-19, mental health, medical students, interim foundation doctors, prospective study

Posted Date: July 7th, 2020

DOl: https://doi.org/10.21203/rs.3.rs-40503/v1

License: (9) (1) This work is licensed under a Creative Commons Attribution 4.0 International License. Read Full License 


\section{Abstract}

Background:

Currently, we can only speculate on what the effects of the COVID-19 pandemic have been on medical students and interim foundation year doctors. In order to support them appropriately both now and, in the future, it is imperative that we understand the impact it has had upon them. This study assessed the effects of the COVID-19 pandemic on medical students and interim foundation year doctors across the United Kingdom (UK), and the support that they received and sought.

Methods:

A prospective, observational, multicentre study was conducted. All medical students and interim foundation year doctors were eligible to participate. The data analysis was carried out as detailed a priori in the protocol.

Findings:

A total of 2075 individuals participated in the SPICE-19 survey from 33 medical schools. There was a significant $(p<0.0001)$ decrease in participants' mood when comparing their mood before the pandemic to during the pandemic. Social distancing and more time at home/with family were the factors that negatively and positively respectively impacted the mood of the greatest number of participants. All areas of life included in the survey were found to have been significantly more negatively impacted than positively impacted $(p<0.0001) .931$ participants wanted more support from their university. Participants were mainly seeking support with exam preparation, course material, and financial guidance.

\section{Discussion:}

Medical and foundation schools need to prepare adequate and effective support. If no action is taken, there may be a knock-on effect on workforce planning and the health of our future workforce. When medical students return to their universities, there is likely to be need for enhanced wellbeing support, adaptations in the short-term and long-term strategies for medical education, and provision of financial guidance.

\section{Introduction}

In January 2020, the World Health Organisation (WHO) declared the outbreak of a novel coronavirus (COVID-19) to be a Public Health Emergency of International Concern ${ }^{1}$. COVID-19 was officially 
characterised as a pandemic in March ${ }^{2}$. As the coronavirus pandemic continues to sweep across the world, there is emerging evidence that the crisis may be generating concern, fear, and stress in the population at large $\mathrm{e}^{3,4}$. These repercussions are likely to result from both the direct effects of COVID-19, as well as the measures imposed to minimise COVID-19 mortality and morbidity ${ }^{5}$. It is important that the effects of this pandemic are acknowledged and characterised as they can have profound effects on individuals, communities, and countries.

Medical students have been particularly affected. They have faced an enormous disruption to their lives ${ }^{6}$. With medical schools having closed ${ }^{7,8}$, students no longer have the structure and stimulation that was previously provided in that environment. They may feel less supported socially and academically by friends and colleagues. At the same time, medical students have been encouraged to take on roles to support national healthcare efforts, which may be especially challenging for students who also hold responsibilities as a parent or caregiver, or who are in high-risk groups. Concerns have been raised that. students who volunteer or graduated early ${ }^{9}$ (interim foundation year 1 (FiY1) doctors) to take on front-line roles may not have sufficient training on infection prevention and control, or sufficient access to adequate personal protective equipment (PPE) ${ }^{10}$ increasing the risk of emotional and/or moral injury ${ }^{11}$. The nature of their roles mean that they may be more exposed to the disease leading to increased concern for their own health and the risk they may pose to others ${ }^{12}$. To mitigate becoming a vector for onward transmission, students and FiY1 doctors may choose to self-isolate ${ }^{13}$. In doing so, they may be less connected with their normal support structures, thereby increasing their risk of depression and anxiety ${ }^{14,15}$. Medical students that take on patient-facing roles may also face stigmatisation ${ }^{16}$ and violence $^{17}$. A significant number may also be living in homes that are detrimental to their physical or mental health.

On the other hand, closure of medical schools may give students more time to focus on past and present hobbies, such as music or art. Many students may feel a sense of pride and fulfilment of moral obligation by taking on roles that support the local, national, or international community ${ }^{18}$. We can only speculate because little formal research exists.

There are no published peer-reviewed articles on the effects the COVID-19 crisis has had on medical students and FiY1 doctors. The Social and Psychological Impact of COVID-19 on medical students: a national survey Evaluation (SPICE-19) study explores the national impact of the COVID-19 pandemic on the social and psychological wellbeing of medical students and FiY1 doctors across the United Kingdom (UK), and the support that they feel they need. Medical students and FiY1 doctors were active 
collaborators at every stage of the research from development, execution, and interpretation to dissemination and translation of the findings.

\section{Methods}

\section{Study design}

This prospective, observational, multicentre cohort study was conducted in line with the pre-specified protocol ${ }^{19}$, using the UK National Research Collaborative Model $^{20}$. This involved inviting medical students and FiY1 doctors to collect real-time data about the mental health and wellbeing of their colleagues in their medical and foundation schools by disseminating an online questionnaire. This study received ethical approval by the University of Oxford Medical Sciences Inter-Divisional Research Ethics Committee (Ethics Approval Reference: R69297/RE001) on 16 ${ }^{\text {th }}$ April 2020.

\section{Setting and Participants}

Any individual that had been enrolled in a medical school in the UK recognised by the General Medical Council (GMC) and listed by the Medical Schools Council (MSC) [Appendix S1] at the start of the 2019/20 academic year was eligible to participate. Anyone below 18 years of age, or unwilling or unable to give informed consent was excluded. Prospective data were collected over four weeks commencing on May $4^{\text {th }} 2020$. Data capture was undertaken using a self-reported, online survey tool on the Qualtrics ${ }^{T M}$ platform. Data from medical students and FiY1 doctors who graduated from one of the eligible 34 medical schools were not collected due to a similar study being conducted locally.

\section{Survey Content}

The survey was co-developed with medical students and FiY1 doctors. It underwent a series of iterations whereby amendments were made, and feedback sought. An initial qualitative data gathering exercise was conducted of 170 medical students or FiY1 doctors that had graduated from the University of Oxford. Based on the concerns and thoughts raised by these individuals, a questionnaire was created. This questionnaire was sent to medical students across the country for their input into its design, content, and usability. Feedback was received from at least one individual at all 34 medical schools in the UK. Based on their experiences, the questionnaire was updated to improve clarity, objectivity, and more accurately capture the needs of our participants during the COVID-19 pandemic. This involved incorporating a pandemic anxiety scale (PAS) [Appendix S2: Section 2, Question 1]; a newly developed measure being utilised by other recently launched studies ${ }^{21,22}$ aiming to characterise the effects of the COVID-19 
pandemic on various sub-groups of the national population. Item 7 of the original PAS (a question related to missing school) was excluded at the time of survey development as it related to a paediatric population. The other eight items were included.

Our final online survey (Appendix S2) had four main components:

1. The demographics of participants: stage of training, ethnicity, age, and gender

2. The wellbeing of participants: pandemic anxiety scale, the mood of participants, and the factors that have had a positive or negative impact on mental health during the COVID-19 pandemic

3. The support offered to participants from their medical school and their foundation school, and the support that participants want

4. Changes to our participants' lives due to the COVID-19 pandemic, and future changes that participants envisage will occur

\section{Statistical methods}

The data was reported using descriptive statistics. Where participants indicated that they would prefer not to answer, they have been removed from the analyses of that section. P values were calculated using two-tailed paired Student's t-test for parametric data. The Mann-Whitney U test or Kruskal-Wallis test with a post-hoc Dunn's Multiple Comparison test were used to calculate $\mathrm{p}$ values for non-parametric data. For Likert scale data for the PAS, a multiple linear regression was utilised. Strongly disagree was given 0 point and strongly agree was given 4 points (with 1,2, and 3 points given to the variables in the middle). The assumptions of the statistical methods used were met. XLSTAT ${ }^{T M} 2020$ statistical software was used.

\section{Results}

\section{Demographics}

Data are provided from 2075 individuals from 33 medical schools. This represented 97\% (33/34) of the medical schools in the UK recognised by the GMC and listed by the MSC at the start of the 2019/20 academic year, and $5 \%$ of the total UK medical student and FiY1 doctor population. Data were not collected from the $34^{\text {th }}$ medical school at the request of that school. 188 responses were excluded as they did not fulfil our inclusion criteria or provide informed consent (Appendix S3). In total, we had data from 1909 medical students and 166 FiY1 doctors. 
Participants were equally spread across all stages of training (Table 1). The majority of our participants were female (72.6\%) and white (65.2\%).

\begin{tabular}{|c|c|}
\hline Demographics & \\
\hline Stage of training & $\begin{array}{l}\text { Medical Students: } \\
\text { Year } 1 \text { (excluding intercalated year) : } 243 \\
\text { Year } 2 \text { (excluding intercalated year) : } 406 \\
\text { Year } 3 \text { (excluding intercalated year) : } 444 \\
\text { Year } 4 \text { (excluding intercalated year) : } 467 \\
\text { Year } 5 \text { (excluding intercalated year) : } 345 \\
\text { FiY1 doctors: } 166 \\
\text { Prefer not to answer: } 4\end{array}$ \\
\hline Age & $\begin{array}{l}\text { Median Age: } 22(\mathrm{IQR}=20-24) \\
\text { Mean Age: } 22.2(\mathrm{~s} . \mathrm{d}=3.4)\end{array}$ \\
\hline Gender & $\begin{array}{l}\text { Male: } 507 \\
\text { Female: } 1507 \\
\text { Non-Binary: } 8 \\
\text { Prefer not to answer: } 53\end{array}$ \\
\hline Ethnicity & $\begin{array}{l}\text { White: } 1352 \\
\text { British: } 1171 \\
\text { Irish: } 34 \\
\text { Other White: } 147 \\
\text { Mixed / Multiple ethnic groups: } 106 \\
\text { White and Black Caribbean: } 11 \\
\text { White and Asian: } 50 \\
\text { White and Black African: } 8 \\
\text { Other Mixed: } 37 \\
\text { Asian/ Asian British: } 427 \\
\text { Indian: } 182 \\
\text { Pakistan: } 87 \\
\text { Bangladeshi: } 16 \\
\text { Chinese: } 66 \\
\text { Other Asian: } 76 \\
\text { Black/ African/ Caribbean/ Black British: } 68 \\
\text { African: } 54 \\
\text { Caribbean: } 9 \\
\text { Other Black: } 5 \\
\text { Other ethnic group: } 40 \\
\text { Prefer not to answer: } 82\end{array}$ \\
\hline
\end{tabular}

Table 1. Demographics of medical students and FiY1 doctors 


\section{Wellbeing of participants}

The mean score on the PAS was $18.9 / 32$. The median score was $19 / 32$ and the interquartile range was $16 / 32$ to 22/32. Of the 1986 participants that completed the PAS, more than half of all participants agreed with five of the PAS statements: 'I think COVID-19 is a very serious issue' (1908, 96.1\%); 'I am worried that I will catch COVID19' (1074, 54.1\%); 'I am worried that friends and family will catch COVID-19'(1804, 90.1\%); 'I am worried that I might transmit the infection to someone else' (1620, 81.6\%); and 'I am worried about the long-term impact this will have on my job prospects and the economy' (1320,66.5\%). Being an intercalated student was the only demographic factor that was significantly associated with a lower PAS score than average $(p=0.003)($ Appendix S4). No demographic factor was significantly associated with a higher PAS score than average.

Overall, participants reported a drop in their mood following the onset of the pandemic $(\mathrm{p}<0.0001)$. Both medical students and FiY1 doctors were similarly affected. FiY1 doctors were more likely to report a positive influence on their mood due to opportunities for paid work $(\mathrm{p}<0.0001)$ (Table 2). With regards to negative influences on mood, cancelled electives negatively affected mood among year 4 and 5 medical students and FiY1 doctors $(\mathrm{p}<0.0001)$; reduction in research opportunities predominantly affected year 4 and 5 medical students ( $p<0.0001)$; and strain of wearing protective equipment chiefly affected FiY1 doctors $(p<0.0001)$. The remaining factors had a similar impact across the different stages of training. Of those who reported experiencing stigma, most had done so for being an individual working in a medical field, but 42 participants reported negative discrimination due to their race. Of these 42 individuals, 31 were of Asian/Asian British ethnicity or White and Asian ethnicity. Although not in the initial list of factors being surveyed, changes to and uncertainty related to medical education and examinations was the most common factor $(n=62)$ cited by individuals in the free text box as a factor negatively influencing mood.

The majority of participants $(n=1886)$ indicated that staying connected with friends/relatives using online tools (1595, 84.6\%), exercise (1590, 84.3\%), watching movies or TV series (1532, 81.2\%), listening to music (1447, 76.7\%), cooking/ baking (1254,66.5\%), and reading books $(1134,60.1 \%)$ to have helped with their mental wellbeing. Other hobbies, faith, meditation, and mental health apps were reported in the free text as being helpful by 183 participants. 


\begin{tabular}{|l|l|}
\hline Factors that had a positive influence & $\begin{array}{l}\text { \% study total who report a positive influence on } \\
\text { mood }(n=/ 1916)\end{array}$ \\
\hline More time at home/ with family & $64.2(1231)$ \\
\hline $\begin{array}{l}\text { Participant and family members remained unaffected } \\
\text { from COVID19 }\end{array}$ & $54.9(1052)$ \\
\hline Reduced responsibilities/ more free time & $54.1(1037)$ \\
\hline Time away from work/university & $46.9(899)$ \\
\hline Cancelled/ Open book exams & $41.3(792)$ \\
\hline Opportunities for paid work & $30.0(577)$ \\
\hline Online learning & $21.5(411)$ \\
\hline Improvements in physical health & $21.0(403)$ \\
\hline Improved financial status & $13.2(253)$ \\
\hline Volunteering opportunities & $11.7(224)$ \\
\hline Increase in research opportunities & $4.1(79)$ \\
\hline Other factors & $3.8(72)$ \\
\hline Factors that had a negative influence on mood & $\%$ study total who report a negative influence on \\
& mood (n) /1916) \\
\hline Social distancing & $77.8(1489)$ \\
\hline Reports on social media and news outlets & $67.1(1285)$ \\
\hline Self-isolation & $61.6(1181)$ \\
\hline Holiday cancelled & $52.0(996)$ \\
\hline Social exclusion & $41.4(793)$ \\
\hline Financial worries & $30.5(584)$ \\
\hline Career uncertainty & $26.1(501)$ \\
\hline Getting infected & $23.7(455)$ \\
\hline Relatives or friends getting infected & $20.7(397)$ \\
\hline Elective cancelled & $16.2(311)$ \\
\hline Deterioration of physical health & $13.0(249)$ \\
\hline Reduction in research opportunities & $12.4(237)$ \\
\hline Strain of wearing protective equipment & $8.5(162)$ \\
\hline High demands in the work setting & $8.2(157)$ \\
\hline Stigmatisation & $7.1(136)$ \\
\hline $\begin{array}{l}\text { Recent bereavement of someone you know from } \\
\text { CovID-19 }\end{array}$ & $6.6(126)$ \\
\hline Other factors & $17.0(326)$ \\
\hline
\end{tabular}

Table 2. Factors affecting mood in medical students and FiY1 doctors.

\section{Support provided to participants}


Over three quarters of participants (1505/1884) reported that their university had provided some form of support. Of the $1505,75.0 \%(n=1128)$ reported that the support had been useful; the three most common forms of support that individuals described as useful were: written support ( $\mathrm{n}=860$ ); support with course material (n = 706); and online material/videos for self-support $(\mathrm{n}=690)$. In the free text box, 196 participants reported support was not useful if there was too much information or if it was too vague to relate to their current situation. Approximately half of the participants (931/1884) reported that they would like more support from their university. The five most common areas of support sought by our participants from their medical schools were: support with exam preparation $(n=569)$; support with course material $(n=531)$; financial guidance $(n=$ 339); online face-to-face support $(\mathrm{n}=323)$; and online material/videos for self-support $(\mathrm{n}=297)$. The majority of medical student participants requesting further support from medical schools $(\mathrm{n}=891)$ were getting teaching from their medical schools $(674,75.6 \%)$. Answers in the free text box highlighted that 108 participants requests for further support entailed either more frequent communication, more transparency, or more empathy for individual personal circumstances. Similarly, 38.9\% (56/144) of FiY1 doctors reported that their foundation school had provided some form of support. Of these 56 FiY1 doctors, 47 found the support useful and 6 indicated they would like more support. Of the 88 FiY1 doctors that reported that they had not received support from their foundation school, a majority $(54,61.4 \%)$ wanted support. They principally wanted written support $(\mathrm{n}=38)$, online face-to-face support $(\mathrm{n}=18)$ or online material/videos for self-support $(\mathrm{n}=18)$.

A minority of participants: $42.7 \%(748 / 1004)$ felt that they had received sufficient information on PPE although the proportion of FiY1 doctors reporting that they had been appropriately informed was higher (76, 53.1\%). Of those who had received sufficient information, their NHS trust was the most commonly cited source of that information (417, 55.7\%). A majority of participants: 56.4\% (975/1729) felt that they had been provided with sufficient training on infection prevention and control. Of the 975 who felt that they had received sufficient information, their university was the most commonly cited source of that information (682, 69.9\%).

\section{Changes experienced by participants}


Figure 1 details the areas of life that participants report to have been affected either positively or negatively by the COVID-19 pandemic. All areas of life surveyed were found to have been significantly more negatively impacted than positively impacted. In the free text box, 118 participants reported that relationships with family and friends was another area of life that had been affected either negatively $(n=19)$ or positively $(n=99)$. Of 1835 participants, all but 8 reported that they more likely than not to continue pursuing a career in medicine.

\section{Conclusions}

\section{Key Findings}

Our findings show that nationally, medical students and FiY1 doctors self-report a decrease in their mood since the start of the pandemic. This is consistent with findings from a single centre-study in $\mathrm{China}^{23}$. A majority report that 'social distancing', 'reports on social media and news outlets', 'self-isolation', and 'cancelled holiday' have had a negative impact on mood. However, there have been factors that have had a positive impact on mood. For the majority of people, these factors included: more time at home/with family; them and their family members remaining unaffected from COVID-19; and reduced responsibility/ more free time. In fact, staying connected with family and friends was reported to be the most frequent method used by participants to help their mental wellbeing. The negative impact of COVID-19 on participants' lives and their mental wellbeing might explain why nearly half of participants indicated that they would like more support from their universities and, to a lesser extent, their foundation schools.

Medical students and doctors are known to suffer with increased rates of anxiety, feelings of pressure, and burnout ${ }^{24,25}$. In order to protect this already at-risk group efforts need to be made to mitigate the detrimental effects the COVID-19 pandemic has had on the overall mental health and wellbeing of medical students and FiY1 doctors. If no action is taken concerns have been raised that fewer medical students may decide to progress immediately to clinical training ${ }^{26}$, or more doctors may choose to take a FY3 year rather than enter a speciality post. Furthermore, there may be a deleterious increase in the number of medical students and FiY1 doctors who develop anxiety, depression, or self-injurious behaviours (such as self-harm and suicide) in excess of the norm in a pre-COVID-19 era. This is evidenced by reports on the psychosocial effects of similar widespread infections, such as the Severe Acute Respiratory Syndrome (SARS) epidemic in $2003^{14,27,28}$. To alleviate the risk of spreading the virus, this support could be provided virtually; internet based psychotherapy has shown similar effectiveness as face-to-face therapy ${ }^{29}$. 
Social distancing, reports on social media and news outlets, and self-isolation were the top three factors that negatively impacted the mood of the greatest number of participants. Therefore, there may be a public health argument to be made about easing the measures seeking to minimise the spread of COVID19 in order to protect the mental health of the population. As the UK government eases restrictions ${ }^{30}$ and increases accessibility of a wider array of COVID-19 tests ${ }^{31}$, it remains to be seen if the effects of social distancing and self-isolation on the mental wellbeing of medical students and FiY1 doctors were temporary or more permanent in nature.

At present there is little guidance as to how best to tackle the effect of media coverage of the pandemic on mental health. The media is critical for providing information. However, misinformation and conspiracy theories spread quickly on social media and can be linked to an increase in anxiety and xenophobia ${ }^{32}$, which was the primary form of stigmatisation reported by our participants. Medical students may be well advised to limit their consumption of news media to trustworthy sources or those news outlets focussing on health promoting behaviours on a time-limited basis.

The most common requests by our participants were for further support related to exam preparation and course materials from their medical schools. UK medical schools are already beginning to employ successful strategies employed for the remote delivery of medical school teaching during the SARS epidemic, such as providing PowerPoint Presentations with lecturers' voice notes ${ }^{33}$, online simulation teaching ${ }^{34}$, and the utilisation of virtual patients ${ }^{35}$. The Medical Schools Council has brought together a range of materials from across UK medical schools to support student learning ${ }^{36}$. It is important to note that there are concerns that the move to virtual teaching could lead to less empathic learners ${ }^{37}$ and reduce the opportunity to acquire practical skills. While the impact on clinical experience is a predominant concern, one cannot overlook the fact that a reduction in research opportunities was also reported as adversely affecting the mood of our participants, especially those in their final two years of medical school. The decreased opportunity for individuals to broaden their skill sets or become more competitive applicants for future careers ${ }^{38}$ may change the trajectory of our future workforce at a time when the clinical academic workforce is already in decline ${ }^{37}$.

Nearly one in ten of our participants reported a recent COVID-related bereavement of someone they knew. While small in number, bereavement can have a profound effect on an individual's wellbeing ${ }^{39}$ and the support that they need, especially for a population group that may not have encountered death. Most of our participants were worried about a friend or family member catching the virus, and a second wave of 
COVID-19 infections may cause a concurrent rise in mortality and morbidity. Therefore, we believe it is critical that medical and foundation schools use this time before a possible second peak to develop necessary and sufficient pastoral support services to support students who have experienced the loss of someone they know.

In addition, almost a third of our participants reported a negative impact on finances due to the pandemic and a lower mood due to this. The association between financial difficulties and adverse mental health outcomes among students has long been noted 40,41 . It is likely that there will be a significant rise in those seeking financial support in order to continue their studies, particularly in those from lower socioeconomic groups. Students are likely to require regular provision of information and support around financing throughout and after the pandemic ${ }^{40,42}$.

\section{Limitations}

The findings of this interim paper assess the impact of COVID-19 limited to a period of national lockdown. These results might indicate important but short-lived reactions of our participants to the changes that have had to be made to teaching and/or the experience of working as a healthcare professional during these unprecedented times. We will be conducting a follow-up study in three months by which time knowledge, provisions, and regulations may have changed. This will allow us to better map the longer-lasting effects of the COVID-19 pandemic on the future of the medical workforce. Secondly, this study was conceived and conducted principally by members of a neurology and neurosurgery interest group. This may mean that dissemination strategies targeted a specific subset of students and FiY1 doctors disproportionately. However, the wide variety of demographic characteristics of our 2000 participants $-5 \%$ of the total population - suggests that our sample is representative of the population in question. Thirdly, our study did not use a validated wellbeing measure. This decision was made in response to feedback from students during the survey development. Priority was given to those areas identified as most relevant by students. The final limitation is that three times as many females as males participated in this study. Females have outnumbered males in medical schools for over 25 years ${ }^{43}$ and there is evidence to suggest that women are more likely to take part in research studies ${ }^{44}$. It may also be because men are less likely to engage in discussions about mental health ${ }^{45}$ which could mean that this study may not be capturing the views of population sub-groups less likely to seek support ${ }^{46}$.

\section{Conclusion}

Despite its limitations, this study offers much needed data to assist in the development and implementation of strategies to protect and prevent mental health issues. The prospective study design 
enables dynamic ascertainment of participants as they live through the COVID-19 pandemic, making the data especially pertinent. Widescale participation of medical students across the UK has allowed for the effects of the pandemic to be studied on a national scale, with statistically significant results. To the authors' knowledge, this is the first national study of medical students' and FiY1 doctors' mental wellbeing and support needs following the COVID-19 pandemic. These interim results, therefore, may have regional, national, and international implications. These data could be used to assist medical schools, foundation schools, and hospital trusts in decision and policy making, signpost medical schools to the most affected student groups, and facilitate the developments of interventions designed to obviate the impact of the pandemic on mental health and wellbeing.

\section{Declarations}

\section{Availability of data and materials}

The dataset supporting the conclusions of this article is included within the article and its additional files.

\section{Ethics approval and consent to participate}

This study received ethical approval by the University of Oxford Medical Sciences Inter-Divisional Research Ethics Committee (Ethics Approval Reference: R69297/RE001) on 16 ${ }^{\text {th }}$ April 2020. All participants provided informed consent.

\section{Consent for publication}

All authors have approved the final manuscript and are willing to take responsibility for appropriate portions of the content.

\section{Competing interests}

KEAS is supported by the Oxford Health NIHR Biomedical Research Centre. The views expressed are those of the authors and not necessarily those of the NHS, the NIHR or the Department of Health. 


\section{Funding}

This research has received no specific grant from any funding agency in the public, commercial, or notfor-profit sectors.

\section{Author contributions}

SB and IG conceived the project. SB, IG, and KEAS contributed equally to the design of the project. SB, IG, $B B, C S G$, and MTB drafted the manuscript. KEAS, AH, and MdAC helped draft and extensively reviewed the manuscript.

\section{Collaborators}

Maria Georgiou, Shumail Mahmood, Anna Casey, Rosalind Di Traglia, Rohan Pancharatnam, Alex Fung, Jack Wellington, Adam Hounat, Jay J. Park, Joshua Erhabor, Mohammad H Ashraf, Hanya Ghazi, Lucas M Hernandez, Zeluleko Sibanda, Makinah Haq, Salma Mahmood, Abbey Boyle, Carlos M. Curtis-Lopez, Harry James Carr, Lorcán McMullan, Michael McLarnon, Emily Bligh, Armin Nazari, Emma Jane Norton, Connor Coyle, Guan Hui Tricia Lim, and Oliver Rushworth

\section{Acknowledgements}

Harry Claireaux for their help with the pilot data gathering exercise.

\section{References}

$1 \quad$ World Health Organization (WHO). Coronavirus disease (COVID-19) outbreak. https://www.who.int/westernpacific/emergencies/covid-19 (accessed April 11, 2020).

2 Mahase E. Covid-19: WHO declares pandemic because of 'alarming levels' of spread, severity, and inaction. BMJ 2020; 368: $\mathrm{m} 1036$. 
3 Brooks SK, Webster RK, Smith LE, et al. The psychological impact of quarantine and how to reduce it: rapid review of the evidence. Lancet. 2020; 395: 912-20.

$4 \quad$ Wang C, Pan R, Wan X, et al. Immediate Psychological Responses and Associated Factors during the Initial Stage of the 2019 Coronavirus Disease (COVID-19) Epidemic among the General Population in China. Int J Environ Res Public Health 2020; 17: 1729.

$5 \quad$ Mahase E. Covid-19: UK starts social distancing after new model points to 260000 potential deaths. BMJ 2020; 368: $\mathrm{m} 1089$.

6 Representatives of the STARSurg Collaborative, EuroSurg Collaborative, and TASMAN Collaborative. Medical student involvement in the COVID-19 response. Lancet (London, England) 2020; published online April 2. DOI:10.1016/S0140-6736(20)30795-9.

7 Medical Schools Council. Advice from Medical Schools Council to UK Medical Schools on actions surrounding Covid-19. .

$8 \quad$ Mahase E. Covid-19: Portugal closes all medical schools after 31 cases confirmed in the country. BMJ. 2020; 368: m986.

9 General Medical Council. Early provisional registration for final year medical students. https://www.gmc-uk.org/news/news-archive/early-provisional-registration-for-final-year-medical-students (accessed June 13, 2020).

10 N. O, T. T, K. F, et al. 'Birdlime' technique using TachoSil tissue sealing sheet soaked with fibrin glue for sutureless vessel transposition in microvascular decompression: Operative technique and nuances. J Neurosurg 2018; 128: 1522-9.

11 Murray E. Moral injury and paramedic practice. J Paramed Pract 2019; 11: 424-5.

12 International Federation of Red Cross and Red Crescent Societies (IFRC). Mental Health and Psychosocial Support for Staff, Volunteers and Communities in an Outbreak of Novel Coronavirus. https://reliefweb.int/report/world/mental-health-and-psychosocial-support-staff-volunteers-andcommunities-outbreak-novel (accessed April 11, 2020).

13 Rose C. Am I part of the cure or am I part of the disease? Keeping coronavirus out when a doctor comes home. N. Engl. J. Med. 2020; 382: 1684-5.

14 Hawryluck L, Gold WL, Robinson S, Pogorski S, Galea S, Styra R. SARS control and psychological effects of quarantine, Toronto, Canada. Emerg Infect Dis 2004; 10: 1206-12.

15 Jeong H, Yim HW, Song YJ, et al. Mental health status of people isolated due to Middle East Respiratory Syndrome. Epidemiol Health 2016; 38: e2016048. 
16 IASC Reference Group on Mental Health and Psychosocial Support in Emergency Settings. Mental Health and Psychosocial Support in Ebola Virus Disease Outbreaks A Guide for Public Health Programme Planners. .

17 McKay D, Heisler M, Mishori R, Catton H, Kloiber O. Attacks against health-care personnel must stop, especially as the world fights COVID-19. Lancet 2020; 0. DOI:10.1016/S0140-6736(20)31191-0.

18 Inter-Agency Standing Committee (IASC). Briefing note on addressing mental health and psychosocial aspects of COVID-19 Outbreak-Version 1.1. .

19 Bandyopadhyay S, Georgiou I, Crespo MDA, Handa A, Saunders K. Social and Psychological Impact of COVID-19 on medical students: a national survey Evaluation Study Protocol. OSF 2020.

20 Bhangu A, Kolias AG, Pinkney T, Hall NJ, Fitzgerald JE. Surgical research collaboratives in the UK. Lancet. 2013; 382: 1091-2.

21 McElroy E, Patalay P, Moltrecht B, et al. Demographic and health factors associated with pandemic anxiety in the context of COVID-19. https://osf.io/jm3vh/?show=view (accessed June 22, 2020).

22 Co-SPACE Study News - Emerging Minds. https://emergingminds.org.uk/co-space-study-news/ (accessed June 12, 2020).

23 Cao W, Fang Z, Hou G, et al. The psychological impact of the COVID-19 epidemic on college students in China. Psychiatry Res 2020; 287. DOI:10.1016/j.psychres.2020.112934.

24 Farrell SM, Kadhum M, Lewis T, Singh G, Penzenstadler L, Molodynski A. Wellbeing and burnout amongst medical students in England. Int Rev Psychiatry 2019; 31: 579-83.

25 Bhugra D, Sauerteig SO, Bland D, et al. A descriptive study of mental health and wellbeing of doctors and medical students in the UK. Int Rev Psychiatry 2019; 31: 563-8.

26 The BMJ. Covid-19-the impact on our medical students will be far-reaching. https://blogs.bmj.com/bmj/2020/04/03/covid-19-the-impact-on-our-medical-students-will-be-farreaching/ (accessed June 22, 2020).

27 Tsang HWH, Scudds RJ, Chan EYL. Psychosocial impact of SARS [2]. Emerg. Infect. Dis. 2004; 10: $1326-7$.

28 Nickell LA, Crighton EJ, Tracy CS, et al. Psychosocial effects of SARS on hospital staff: Survey of a large tertiary care institution. CMAJ 2004; 170: 793-8.

29 Barak A, Hen L, Boniel-Nissim M, Shapira N. A Comprehensive Review and a Meta-Analysis of the Effectiveness of Internet-Based Psychotherapeutic Interventions. J Technol Hum Serv 2008; 26: 109-60. 
30 Our plan to rebuild: The UK Government's COVID-19 recovery strategy - GOV.UK. https://www.gov.uk/government/publications/our-plan-to-rebuild-the-uk-governments-covid-19-recoverystrategy (accessed June 12, 2020).

31 Testing for COVID-19 antibodies in frontline healthcare workers.

https://medicalxpress.com/news/2020-06-covid-antibodies-frontline-healthcare-workers.html (accessed June 12, 2020).

32 The pandemic of social media panic travels faster than the COVID-19 outbreak.

https://www.ncbi.nlm.nih.gov/pmc/articles/PMC7107516/ (accessed June 12, 2020).

33 Schultze-Mosgau S, Zielinski T, Lochner J. Interactive, web-based e-lectures with a multimedia online examination. Med Educ 2004; 38: 1184.

34 Abrahamson SD, Canzian S, Brunet F. Using simulation for training and to change protocol during the outbreak of severe acute respiratory syndrome. Crit Care 2005; 10: R3.

35 Lim ECH, Ong BKC, Seet RCS. Using videotaped vignettes to teach medical students to perform the neurologic examination [2]. J. Gen. Intern. Med. 2006; 21: 101.

36 Medical Schools Council. Information for medical students.

https://www.medschools.ac.uk/covid-19/information-for-medical-students (accessed June 22, 2020).

37 Medical Research Council. OSCHR UK-Wide Survey of Health Research Fellowships 2009. https://mrc.ukri.org/publications/browse/oschr-uk-wide-survey-of-health-research-fellowships-2009/ (accessed June 19, 2020).

38 Taylor AK, Purdy S. How to get involved in research as a medical student. BMJ 2017; 356: i6593.

39 Parkes CM. Coping with loss: Bereavement in adult life. BMJ 1998; 316: 856.

40 Richardson T, Elliott P, Roberts R, Jansen M. A Longitudinal Study of Financial Difficulties and Mental Health in a National Sample of British Undergraduate Students. Community Ment Health J 2017; 53: $344-52$.

41 Pisaniello MS, Asahina AT, Bacchi S, et al. Effect of medical student debt on mental health, academic performance and specialty choice: A systematic review. BMJ Open. 2019; 9.

DOI:10.1136/bmjopen-2019-029980.

42 Ross S, Cleland J, Macleod MJ. Stress, debt and undergraduate medical student performance. Med Educ 2006; 40: 584-9.

43 Moberly T. Number of women entering medical school rises after decade of decline. Bmj 2018; 360: 167-167. 
44 Smith G, Smith WG. Does gender influence online survey participation?: A record-linkage analysis of university faculty online survey response behavior. 2008.

45 McKenzie SK, Collings S, Jenkin G, River J. Masculinity, Social Connectedness, and Mental Health: Men's Diverse Patterns of Practice. Am J Mens Health 2018; 12: 1247-61.

46 Addis ME, Mahalik JR. Men, Masculinity, and the Contexts of Help Seeking. Am. Psychol. 2003; 58: 5-14.

\section{Figures}

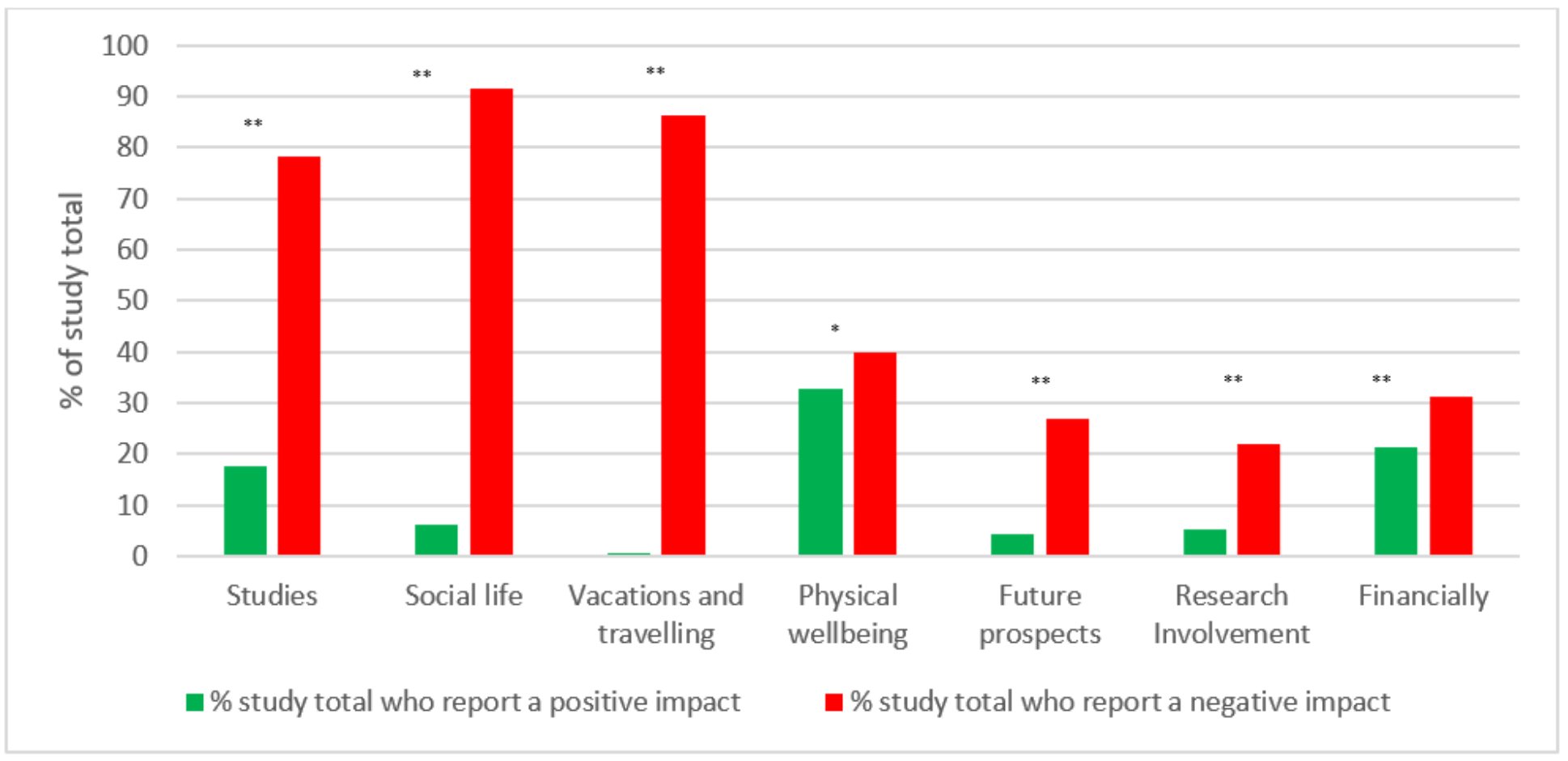

Figure 1

Areas of life affected by COVID-19 according to medical students and FiY1 doctors. These values do not total $100 \%$ as some participants did not report a positive or negative impact to that area of their life due to COVID-19. * $P$ value $<0.001 * * P$ value $<0.0001$

\section{Supplementary Files}

This is a list of supplementary files associated with this preprint. Click to download.

- Appendix.docx

- AppendixS4.png 